\title{
Flat conformal structures and the classification of de Sitter manifolds
}

\begin{abstract}
Kevin P. Scannell
Given a compact $n$-manifold $\Sigma$ with a flat conformal structure, there is a canonical procedure for constructing an associated $(n+$ 1)-dimensional de Sitter spacetime homeomorphic to $\Sigma \times(0, \infty)$; we call these standard de Sitter spacetimes. Our main theorem is a classification of compact de Sitter manifolds; it asserts that every de Sitter spacetime which is a small regular neighborhood of a closed spacelike hypersurface isometrically embeds in a standard de Sitter spacetime. This complements results of G. Mess in the flat and anti-de Sitter cases.
\end{abstract}

\section{Introduction.}

A spacetime is a connected, smooth $n$-manifold with a metric of Lorentzian signature $(n-1,1)$. For simplicity we will restrict our attention to spacetimes which are orientable and time-orientable. A de Sitter (resp. flat, anti-de Sitter) spacetime is a spacetime of constant positive (resp. zero, negative) curvature. Mess [23] has classified all compact $(2+1)$-dimensional flat and anti-de Sitter spacetimes which are domains of dependence (see $\S 3$ ), answering a question of Witten [29]. Our main theorem extends the classification to the de Sitter case.

The paper of Mess relies on the techniques of [4], in which it is shown that a closed flat spacetime is geodesically complete. Klingler [17] has recently generalized this argument to work for all constant curvature spacetimes (see also [24]). This allows one to deduce, for instance, that there are no closed de Sitter spacetimes (because no infinite group of isometries acts discontinuously on de Sitter space $[30, \S 11.1])$. The groups of isometries acting cocompactly on flat Minkowski space or anti-de Sitter space have been widely studied; see [8], [12], [10], [21] for more information.

In this paper, we study the case of compact spacetimes with non-empty, spacelike boundary. These are called spacetime-bordisms, viewed as bordisms between their past and future boundary components. Our general 
approach to classifying spacetime-bordisms is to first describe those which are domains of dependence (in particular these are topologically products $\Sigma \times \mathbf{R}$ with spacelike slices). One would then like to show that an arbitrary spacetime-bordism of constant curvature is in fact a domain of dependence, as in [23]. Our main theorem completes the first part of this program in the remaining case of de Sitter spacetime-bordisms (Theorem 1.1). It turns out, however, that there are simple examples of de Sitter spacetime-bordisms which are not domains of dependence; these are discussed in the final section.

To state our classification theorem, we need a construction due to Thurston. Suppose $\Sigma$ is a compact $n$-manifold without boundary, equipped with a flat conformal (Möbius) structure. In unpublished work, Thurston has shown how to "thicken" a developing map dev $: \tilde{\Sigma} \rightarrow \mathbf{S}^{n}$ of the flat conformal structure to obtain an equivariant immersion $D: \tilde{\Sigma} \times(0, \infty) \rightarrow \mathbf{H}^{n+1}$, and hence a hyperbolic metric on $\Sigma \times(0, \infty)$. In dimension two, this was used by Thurston to parameterize $\mathbf{C} P^{1}$-structures on $\Sigma$ by the space of measured geodesic laminations on $\Sigma$ (these arise as "bending laminations" on the frontier of the image of $D$ ). The projective dual of this construction provides an equivariant immersion of $\tilde{\Sigma} \times(0, \infty)$ into $(n+1)$-dimensional de Sitter space, inducing a de Sitter metric on $\Sigma \times(0, \infty)$; the spacetimes obtained in this way are the standard de Sitter spacetimes, constructed in detail in $\S 5$.

Theorem 1.1. Every de Sitter spacetime which is a small regular neighborhood of a compact spacelike hypersurface isometrically embeds in a standard de Sitter spacetime.

The first two sections contain background material on geometric structures $(\S 2)$ and causality in Lorentzian manifolds $(\S 3)$. We proceed to describe the canonical decomposition of a flat conformal manifold ( $\S 4)$, and how it gives rise to a standard de Sitter spacetime $(\S 5)$. This is followed by some convexity properties in constant curvature spacetimes which yield our main result; many of these results work in the flat and anti-de Sitter cases, simplifying some of the arguments in [23]. We conclude with a brief discussion of when a de Sitter spacetime-bordism is a domain of dependence.

I would like to thank my thesis advisor Geoffrey Mess for his support and many helpful discussions concerning this work. Thanks also to Robert Greene, Lai-Sang Young, and Bob Edwards. 
Flat conformal structures and the classification of de Sitter manifolds 327

\section{Geometric Structures and Deformation Spaces.}

Good references for the material presented in this section are [3], [11], and [28]. Suppose $G$ is a Lie group which acts faithfully, transitively, and analytically on a manifold $X$. Let $M$ be a connected $C^{0,1}$ manifold, possibly with boundary, with a fixed basepoint $m_{0} \in M$. By convention, the universal cover a space will always be indicated by the addition of a tilde, so $\tilde{M}$ denotes the universal cover of $M$.

A based $(G, X)$-structure on $M$ is a pair $(f, \phi)$ consisting of a $C^{0,1}$ local embedding $f: \tilde{M} \rightarrow X$, and a homomorphism $\phi: \pi_{1}\left(M, m_{0}\right) \rightarrow G$ satisfying:

$$
f(\gamma \cdot x)=\phi(\gamma) \cdot f(x)
$$

for all $\gamma \in \pi_{1}\left(M, m_{0}\right)$ and all $x \in \tilde{M}$ (we say $f$ is $\phi$-equivariant). The homomorphism $\phi$ is called the holonomy representation of the based $(G, X)$ structure, and $f$ is called the developing map. Let $\mathcal{D}_{(G, X)}(M)$ denote the set of based $(G, X)$-structures on $M$, identifying pairs which differ by the action of a diffeomorphism $g:\left(M, m_{0}\right) \rightarrow\left(M, m_{0}\right)$ isotopic to the identity rel $m_{0}$. The deformation space $\mathcal{T}_{(G, X)}(M)$ of $(G, X)$-structures on $M$ is defined to be the quotient of $\mathcal{D}_{(G, X)}(M)$ under conjugation by $G$. A $(G, X)$ manifold is a pair consisting of a connected, $C^{0,1}$ manifold $M$ and a point in $\mathcal{T}_{(G, X)}(M)$. We will habitually abuse terminology by referring to an element of $\mathcal{T}_{(G, X)}(M)$ by a representative based $(G, X)$-structure.

Our primary examples of geometric structures will come from the constant curvature Riemannian and Lorentzian model spaces. Fix integers $0 \leq k \leq n$ with $n \geq 2$, and define $\mathbf{R}_{k}^{n}$ to be the space $\mathbf{R}^{n}$ equipped with the signature $(n-k, k)$ inner product

$$
\langle\mathbf{v}, \mathbf{w}\rangle=-\sum_{i=1}^{k} v_{i} w_{i}+\sum_{j=k+1}^{n} v_{j} w_{j} .
$$

When $k=1$, we call $\mathbf{R}_{1}^{n}$ (flat) Minkowski space. Recall that a vector $\mathbf{v} \in \mathbf{R}_{1}^{n}$ is said to be:

- spacelike if $\langle\mathbf{v}, \mathbf{v}\rangle>0$;

- null or lightlike if $\langle\mathbf{v}, \mathbf{v}\rangle=0$;

- timelike if $\langle\mathbf{v}, \mathbf{v}\rangle<0$;

- causal if $\langle\mathbf{v}, \mathbf{v}\rangle \leq 0$. 
Define:

$$
\mathbf{S}_{1}^{n}=\left\{\mathbf{v} \in \mathbf{R}_{1}^{n+1} \mid\langle\mathbf{v}, \mathbf{v}\rangle=1\right\}
$$

$\mathbf{S}_{1}^{n}$ is our model of $n$-dimensional de Sitter space; it inherits a Lorentzian metric of constant curvature +1 . Note that $\mathbf{S}_{1}^{n}$ is homeomorphic to $\mathbf{S}^{n-1} \times \mathbf{R}$, and admits a natural conformal compactification $\overline{\mathbf{S}_{1}^{n}} \approx \mathbf{S}^{n-1} \times[0,1]$ by $(n-1)$-spheres $\partial_{\infty}^{-} \mathbf{S}_{1}^{n}$ and $\partial_{\infty}^{+} \mathbf{S}_{1}^{n}$ at past and future infinity respectively.

An alternative model of de Sitter space is constructed by means of the natural projection $\varpi: \mathbf{R}_{1}^{n+1} \backslash\{0\} \rightarrow \mathbf{R} P^{n}$. Define $\left(\mathbf{H}^{n}\right)^{*}$ to be the image in $\mathbf{R} P^{n}$ of the spacelike vectors of $\mathbf{R}_{1}^{n+1}$; we call $\left(\mathbf{H}^{n}\right)^{*}$ the projective model of de Sitter space. Recall that the image in $\mathbf{R} P^{n}$ of the timelike vectors of $\mathbf{R}_{1}^{n+1}$ is the usual projective (Klein) model of $n$-dimensional hyperbolic space $\mathbf{H}^{n}$, with the projectivized null vectors corresponding to the sphere at infinity $\partial_{\infty} \mathbf{H}^{n}$; thus $\partial_{\infty} \mathbf{H}^{n}$ simultaneously compactifies $\mathbf{H}^{n}$ and $\left(\mathbf{H}^{n}\right)^{*}$. The advantage of this model is that we may exploit the projective duality between $k$-planes in $\mathbf{H}^{n}$ and $(n-k-1)$-planes in $\left(\mathbf{H}^{n}\right)^{*}$ to transfer certain standard constructions from hyperbolic space to de Sitter space (compare $[5],[15],[26])$.

In light of the above discussion, we will be considering families of $(G, X)$-manifolds with $G=S O_{0}(n, 1)$, the identity component of $O(n, 1)$. This group is simultaneously isomorphic to the group $\operatorname{Isom}^{+}\left(\mathbf{H}^{n}\right)$ of orientation-preserving isometries of $\mathbf{H}^{n}$, the group $M \ddot{o} b^{+}\left(\mathbf{S}^{n-1}\right)$ of orientation-preserving Möbius transformations of $\mathbf{S}^{n-1}$, and the group $\operatorname{Isom}_{\uparrow}^{+}\left(\mathbf{S}_{1}^{n}\right)$ of orientation-preserving, orthochronous isometries of $\mathbf{S}_{1}^{n}$. Corresponding to these three identifications, we have the following examples of $(G, X)$-structures on an orientable $n$-manifold:

- A hyperbolic structure is an $\left(S_{0}(n, 1), \mathbf{H}^{n}\right)$-structure. The existence of a hyperbolic structure on an orientable $n$-manifold is equivalent to the existence of a Riemannian metric of constant negative curvature.

- A flat conformal structure is an $\left(S O_{0}(n+1,1), \mathbf{S}^{n}\right)$ structure. In dimension two this is simply the classical notion of a projective or $\mathbf{C} P^{1}$ structure on a Riemann surface. For $n \geq 3$, Liouville's Theorem states that a conformal diffeomorphism of domains in $\mathbf{S}^{n}$ is the restriction of a Möbius transformation. It follows that a flat conformal structure is equivalent to a (locally) conformally flat Riemannian metric [18], [22].

- A de Sitter structure is an $\left(S O_{0}(n, 1), \mathrm{S}_{1}^{n}\right)$-structure. 
Flat conformal structures and the classification of de Sitter manifolds 329

We will use the abbreviations $\mathbf{H}^{n}(M), \mathcal{C}(M)$, and $\mathbf{S}_{1}^{n}(M)$ for the respective deformation spaces $\mathcal{T}_{(G, X)}(M)$ of hyperbolic, flat conformal, and de Sitter structures on an $n$-manifold $M$.

\section{Elementary Causality.}

Let $\mathcal{M}$ be a spacetime, and consider a point $x \in \mathcal{M}$. Define $I^{+}(x)$ to be the set of points $p \in \mathcal{M}$ such that there exists a non-trivial past-pointing timelike curve from $p$ to $x$ (when $\mathcal{M}=\mathbf{S}_{1}^{n}$, this definition works equally well for points $\left.x \in \partial_{\infty}^{-} \mathbf{S}_{1}^{n}\right)$. If $\Sigma$ is a subset of $\mathcal{M}$, let $I^{+}(\Sigma)=\cup_{x \in \Sigma} I^{+}(x)$. The set $I^{+}(\Sigma)$ is clearly open, and is called the chronological future of $\Sigma$ in $\mathcal{M}$. The chronological past $I^{-}(x)$ is defined by replacing "past-pointing" with "futurepointing" in the definition of $I^{+}(x)$; similarly, define $I^{-}(\Sigma)=\cup_{x \in \Sigma} I^{-}(x)$. The future domain of dependence $D^{+}(\Sigma)$ is defined to be the set of points $p \in \mathcal{M}$ such that every inextendible past-pointing causal curve starting at $p$ intersects $\Sigma$. The future Cauchy horizon is given by

$$
H^{+}(\Sigma)=\overline{D^{+}(\Sigma)} \backslash D^{+}(\Sigma) .
$$

The sets $D^{-}(\Sigma)$ and $H^{-}(\Sigma)$ are defined analogously. We say $\Sigma$ is achronal (resp. acausal) if no timelike (resp. causal) curve intersects $\Sigma$ more than once. Finally, $\Sigma$ is called a global Cauchy hypersurface for $\mathcal{M}$ if it is a closed, spacelike, acausal hypersurface, and $\mathcal{M}=D^{+}(\Sigma) \cup D^{-}(\Sigma)$; when such a hypersurface exists $\mathcal{M}$ is said to be a domain of dependence.

Lemma 3.1. [25, Ch. 14, Lemma 43] If $\Sigma$ is a closed acausal hypersurface, then $D^{+}(\Sigma) \cup D^{-}(\Sigma)$ is open.

If we are given a closed, spacelike, acausal hypersurface $\Sigma \subset \mathcal{M}$, then to show $\Sigma$ is a global Cauchy hypersurface for $\mathcal{M}$ it suffices by this lemma to show that $H^{+}(\Sigma)=H^{-}(\Sigma)=\emptyset$. Showing that the Cauchy horizons vanish is facilitated by the following elementary characterization of $H^{+}(\Sigma)$, which can be assembled from the standard references (e.g. [14, Ch. 6], [25, Ch. 14], [2]).

Lemma 3.2. Suppose $\Sigma$ is a closed acausal hypersurface. Then if $H^{+}(\Sigma)$ is non-empty, it is a closed achronal $C^{0,1}$ hypersurface disjoint from $\Sigma$. Furthermore, a point $x$ is in $H^{+}(\Sigma)$ if and only if the following two conditions hold:

- every inextendible past-pointing timelike curve starting at $x$ intersects $\Sigma$; 
- there exists an inextendible past-pointing null geodesic ray starting at $x$ which lies entirely within $H^{+}(\Sigma)$.

Here and in what follows, our results are stated for the future Cauchy horizon $H^{+}(\Sigma)$, the statements for $H^{-}(\Sigma)$ being completely analogous. The null geodesic rays given by Lemma 3.2 are called the null generators of $H^{+}(\Sigma)$.

We will now specialize the discussion of causality to the special case of hypersurfaces in constant curvature spacetimes. A spacelike de Sitter hypersurface is a compact, oriented, smooth $n$-manifold $\Sigma$ without boundary, equipped with a based $\left(S O_{0}(n+1,1), \mathbf{S}_{1}^{n+1}\right)$-structure $(f, \phi)$ such that $f$ is a spacelike immersion; it follows that $\Sigma$ inherits a well-defined complete Riemannian metric. Similarly there are notions of spacelike flat hypersurface and spacelike anti-de Sitter hypersurface.

Given a spacelike de Sitter hypersurface $\Sigma$, let $\mathcal{M}=\Sigma \times(0, \infty)$ and define $\mathfrak{D}(\Sigma) \subseteq \mathbf{S}_{1}^{n+1}(\mathcal{M})$ to be the set of all de Sitter structures on $\mathcal{M}$ such that there exists an isometric embedding of $\Sigma$ as a global Cauchy hypersurface for $\mathcal{M}$. The set $\mathfrak{D}(\Sigma)$ is partially ordered by inclusion, and we obtain by Zorn's Lemma a maximal element $\mathcal{M}_{\max }(\Sigma)$. From the existence of the developing map, one sees that the "germ of extensions" is unique; i.e. for any two elements $\mathcal{M}_{1}, \mathcal{M}_{2} \in \mathfrak{D}(\Sigma)$ there exist $\mathcal{M}_{3} \in \mathfrak{D}(\Sigma)$ and isometric embeddings of $\mathcal{M}_{3}$ in both $\mathcal{M}_{1}$ and $\mathcal{M}_{2}$. Using this fact and an argument of Choquet-Bruhat and Geroch $[14, \S 7.6])$, one can show that every element of $\mathfrak{D}(\Sigma)$ isometrically embeds in $\mathcal{M}_{\max }(\Sigma)$. We identify $\Sigma$ with its image in $\mathcal{M}_{\max }(\Sigma)$. Again, a similar construction works for the flat and anti-de Sitter cases. The spacetime $\mathcal{M}_{\text {max }}(\Sigma)$ is called the maximal domain of dependence for $\Sigma$. By a result of Geroch [9], it is homeomorphic to $\Sigma \times \mathbf{R}$ in such a way that each slice $\Sigma \times\{t\}$ is a global Cauchy hypersurface.

If $\tilde{\Sigma}$ is the universal cover of a spacelike de Sitter (resp. flat, antide Sitter) hypersurface, one can also define a maximal domain of dependence $\mathcal{M}_{\text {max }}(\tilde{\Sigma})$, which coincides with the universal cover of $\mathcal{M}_{\text {max }}(\Sigma)$. When the future frontier of the developing image is non-empty, it is possible to attach a boundary with nice causal properties to $\mathcal{M}_{\max }(\tilde{\Sigma})$ :

Proposition 3.3. Suppose $\Sigma$ is a spacelike de Sitter hypersurface. Then either $\mathcal{M}_{\text {max }}(\Sigma)$ is future complete or else $\mathcal{M}_{\text {max }}(\tilde{\Sigma})$ embeds in a de Sitter spacetime in which the following conditions hold:

(1) $H^{+}(\tilde{\Sigma})$ is non-empty;

(2) Every null generator of $H^{+}(\tilde{\Sigma})$ is past complete; 
Flat conformal structures and the classification of de Sitter manifolds 331

(3) Every null generator is either future complete or contains a future endpoint.

Proof. Let dev : $\mathcal{M}_{\max }(\tilde{\Sigma}) \rightarrow \mathbf{S}_{1}^{n+1}$ denote the developing map (here we have used the fact that $\mathcal{M}_{\max }(\tilde{\Sigma}) \approx \tilde{\Sigma} \times \mathbf{R}$ is simply-connected). Assume there exists a future incomplete timelike curve in $\mathcal{M}_{\max }(\Sigma)$. The argument for Lemma 3.2 also shows that the future frontier of $\operatorname{dev}\left(\mathcal{M}_{\max }(\tilde{\Sigma})\right)$ is locally a Lipschitz submanifold of $\mathbf{S}_{1}^{n+1}$. In this way, we can form a manifold with boundary $\mathcal{M}^{\prime}=\mathcal{M}_{\max }(\tilde{\Sigma}) \cup H$, equipped with the obvious extension of the developing map. (The set $H$ can also be thought of as the collection of "terminal indecomposable past sets" corresponding to future incomplete timelike rays as in $[14, \S 6.8])$. Because $\mathcal{M}_{\max }(\tilde{\Sigma})$ is a maximal domain of dependence, $H=H^{+}(\tilde{\Sigma})$ in $\mathcal{M}^{\prime}$.

Consider an arbitrary point $x \in H^{+}(\tilde{\Sigma})$ lying on a null generator $\lambda$. Let $\left\{\beta_{j}\right\}$ be a sequence of inextendible past-pointing timelike curves starting at $x$ and approaching $\lambda$. Suppose $\lambda$ is past incomplete, and let $p \in \mathbf{S}_{1}^{n+1}$ be the past endpoint of $\operatorname{dev}(\lambda)$. It follows that only finitely many of the curves $\operatorname{dev}\left(\beta_{j}\right)$ enter $I^{-}(p)$, or else we could construct a timelike curve back in $\mathcal{M}^{\prime}$ corresponding to the missing endpoint of $\lambda$. Thus infinitely many of the $\operatorname{dev}\left(\beta_{j}\right)$ meet $\operatorname{dev}(\tilde{\Sigma})$ before reaching $I^{-}(p)$; this contradicts the completeness of $\tilde{\Sigma}$.

Consider a sequence of points $x_{j}$ which lie on a null generator for $H^{+}(\tilde{\Sigma})$ such that $x_{j}$ is to the past of $x_{j+1}$, and suppose $\operatorname{dev}\left(x_{j}\right) \rightarrow p$. Take a pastpointing timelike segment from each point $x_{j}$, so that the endpoints form a timelike-separated sequence $\left\{z_{j}\right\}$ in $\mathcal{M}_{\max }(\tilde{\Sigma})$ and $\operatorname{dev}\left(z_{j}\right) \rightarrow p$. These points can be joined by a future-pointing timelike curve whose equivalence class is the limit of the $x_{j}$. We conclude that the null generators are closed sets and (3) follows.

When $\mathcal{M}_{\max }(\Sigma)$ fails to be future complete, the spacetime given by Proposition 3.3 will be denoted $\overline{\mathcal{M}}_{\max }(\tilde{\Sigma})$. It should be noted that the above discussion greatly simplifies in the flat and anti-de Sitter cases. For instance, an easy argument shows that in these cases the developing map from the universal cover of a closed spacelike hypersurface is an achronal embedding [13]. The existence of exotic developing maps into $\mathbf{S}_{1}^{n}$ is the main difficulty one encounters is extending the results of [23].

The following elementary proposition will come in handy during the proof of the main theorem. 
Proposition 3.4. Let $\lambda$ be a past complete null ray in $\mathbf{S}_{1}^{n}$ or $\mathbf{R}_{1}^{n}$. Then there is a unique degenerate hyperplane $N$ containing $\lambda$ and $I^{+}(\lambda)=I^{+}(N)$.

Proof. Consider first the case when $\lambda \subset \mathbf{R}_{1}^{n}$; without loss of generality we may assume $\lambda$ is a line through the origin in the direction of some pastpointing null vector $\mathbf{n} \in \mathbf{R}_{1}^{n}$. Let $N=\mathbf{n}^{\perp}$; that is, the subspace

$$
\mathbf{n}^{\perp}=\left\{\mathbf{v} \in \mathbf{R}_{1}^{n} \mid\langle\mathbf{n}, \mathbf{v}\rangle=0\right\} .
$$

It follows easily that $N$ is the unique degenerate hyperplane containing $\lambda$, and that

$$
I^{+}(N)=\left\{\mathbf{v} \in \mathbf{R}_{1}^{n} \mid\langle\mathbf{n}, \mathbf{v}\rangle>0\right\} .
$$

Clearly $I^{+}(\lambda) \subseteq I^{+}(N)$; for the converse, consider a point $\mathbf{w} \in I^{+}(N)$. It suffices to find a point $\mathbf{p} \in \lambda$ with $\langle\mathbf{w}-\mathbf{p}, \mathbf{w}-\mathbf{p}\rangle<0$. We have

$$
\langle\mathbf{w}-t \mathbf{n}, \mathbf{w}-t \mathbf{n}\rangle=\langle\mathbf{w}, \mathbf{w}\rangle-2 t\langle\mathbf{w}, \mathbf{n}\rangle
$$

and $\langle\mathbf{w}, \mathbf{n}\rangle>0$, so choosing $t>0$ large enough gives our desired vector $\mathbf{p}$.

In de Sitter space $\mathbf{S}_{1}^{n}$, the degenerate $k$-planes are precisely the intersections with $\mathbf{S}_{1}^{n}$ of the degenerate $(k+1)$-planes through the origin in $\mathbf{R}_{1}^{n+1}$. Using this remark, the result for de Sitter space follows easily.

\section{The Canonical Stratification.}

Throughout this section, we let $\Sigma$ denote a compact, connected $n$ dimensional manifold without boundary. Let $D_{\infty}: \tilde{\Sigma} \rightarrow \mathbf{S}^{n}$ be a developing map and $\phi$ a holonomy representation for a flat conformal structure on $\Sigma$. A construction originally due to Thurston (unpublished, see [16]) and extended by Kulkarni-Pinkall [19, 20] and Apanasov [1] produces a canonical decomposition of $\Sigma$ with respect to this structure. This technique will be used to construct families of hyperbolic and de Sitter structures on $\Sigma \times(0, \infty)$ parameterized by $\mathcal{C}(\Sigma)$. Our presentation follows [20].

We begin by using $D_{\infty}$ to pull back the usual metric on $\mathbf{S}^{n}$ to a metric on $\tilde{\Sigma}$, and considering the metric space completion $\bar{\Sigma}$ of $\tilde{\Sigma}$. There is a unique continuous extension $\bar{D}_{\infty}: \bar{\Sigma} \rightarrow \mathbf{S}^{n}$ of $D_{\infty}$. A subset $U \subset \tilde{\Sigma}$ is an open round ball if $D_{\infty}$ maps $U$ homeomorphically onto an open round ball in $\mathbf{S}^{n}$. Given an open round ball $U$ in $\tilde{\Sigma}$, the closure $\bar{U}$ in $\bar{\Sigma}$ maps homeomorphically to a 
Flat conformal structures and the classification of de Sitter manifolds 333

closed round ball in $\mathbf{S}^{n}$, hence $\bar{U}$ is conformally equivalent to compactified hyperbolic space $\mathbf{H}^{n} \cup \partial_{\infty} \mathbf{H}^{n}$. We may therefore transfer the usual notion of "hyperbolic convex hull" to $\bar{U}$; let $U_{\infty}=\bar{U} \backslash \tilde{\Sigma}$ and let $C(U)$ denote the intersection of $U$ and the convex hull of $U_{\infty}$ in $\bar{U}$ (note that $C(U)=\emptyset$ if and only if $U_{\infty}$ has fewer than two points).

Proposition 4.1. Exactly one of the following holds:

(1) $\tilde{\Sigma} \cong \mathbf{S}^{n}$ with the obvious flat conformal structure;

(2) $\tilde{\Sigma} \cong \mathbf{E}^{n}=\mathbf{S}^{n} \backslash\{\infty\}$;

(3) For every $p \in \tilde{\Sigma}$, there exists a unique open round ball $U_{p}$ such that $p \in C\left(U_{p}\right)$.

Proof. Fix $p \in \tilde{\Sigma}$, and let $W_{p}$ be the union of all open round balls containing $p$ (this set is non-empty because $D_{\infty}$ is a local diffeomorphism). One checks easily that the restriction of $D_{\infty}$ to $W_{p}$ is injective, because $D_{\infty}$ is injective on the union of any two open round balls meeting in a "spherical lens". Let $F=\mathbf{S}^{n} \backslash D_{\infty}\left(W_{p}\right)$; this set is the intersection of closed round balls in $\mathrm{S}^{n}$, and is therefore a closed convex set. Suppose $F$ has fewer than two points. Then $W_{p}$ is conformally equivalent to either $\mathbf{S}^{n}$ or $\mathbf{E}^{n}$, and if the dimension of $\tilde{\Sigma}$ is at least two it follows that $\tilde{\Sigma}=\overline{W_{p}} \cong \mathbf{S}^{n}$ or $\mathbf{E}^{n}$ (in the one-dimensional case, we obtain the same conclusion without necessarily having $\tilde{\Sigma}=\overline{W_{p}}$ ). We shall assume therefore that $F$ has at least two points and without loss of generality that $D_{\infty}(p)=\infty \in \mathbf{S}^{n}$, so we can view $F$ as a subset of $\mathbf{E}^{n}=\mathbf{S}^{n} \backslash\{\infty\}$. Hence there exists a unique closed round ball $B$ of least radius containing $F$; since $D_{\infty}$ is injective on $W_{p}$, the set $U_{p}=D_{\infty}^{-1}\left(\mathbf{S}^{n} \backslash B\right)$ is an open round ball in $\tilde{\Sigma}$. We claim $p \in C\left(U_{p}\right)$.

By tracing through the definitions, we have that $\bar{D}_{\infty}\left(\left(U_{p}\right)_{\infty}\right)=F \cap \partial B$ and so $p \in C\left(U_{p}\right)$ if and only if $D_{\infty}(p)$ is in the convex hull of $F \cap \partial B$ (taken in the complement of $B$ ). By inversion in $\partial B$, this in turn is equivalent to the Euclidean center of $B$ lying in the convex hull of $F \cap \partial B$ (taken in $B$ ). If this failed to hold however, one could construct a closed round ball of lesser radius containing $F$.

Uniqueness of $U_{p}$ is clear, the cogent remark being that for any pair of open round balls $U_{1}$ and $U_{2}$ in $\mathbf{S}^{n}$, the convex hull of $\partial U_{1} \backslash U_{2}$ in $U_{1}$ and the convex hull of $\partial U_{2} \backslash U_{1}$ in $U_{2}$ must be disjoint.

The flat conformal structure is said to be of elliptic type, parabolic type, or hyperbolic type, depending on whether (1), (2), or (3) holds in the statement 
of Proposition 4.1. In the case of hyperbolic type, the decomposition $\tilde{\Sigma}=$ $\bigcup_{p \in \tilde{\Sigma}} C\left(U_{p}\right)$ is called the canonical stratification of $\tilde{\Sigma}$; each $C\left(U_{p}\right)$ is a called a stratum. The set of strata is written $\mathcal{S}$. Note finally that this decomposition is equivariant with respect to the action of $\pi_{1}(\Sigma)$ on $\tilde{\Sigma}$, and so there is an induced stratification of $\Sigma$.

\section{Standard de Sitter Spacetimes.}

A $C^{1}$ path $\alpha:[0,1] \rightarrow \mathcal{M}$ in a spacetime $\mathcal{M}$ has length defined by

$$
L(\alpha)=\int_{\alpha}|\langle\dot{\alpha}, \dot{\alpha}\rangle|^{\frac{1}{2}}
$$

An admissible spacelike partition (resp. timelike, causal) for a continuous path $\alpha:[0,1] \rightarrow \mathcal{M}$ is a finite partition $0=t_{0}<t_{1}<\cdots<t_{k-1}<t_{k}=1$. such that for every $j \in 0, \ldots, k-1$, the points $\alpha\left(t_{j}\right)$ and $\alpha\left(t_{j+1}\right)$ can be joined by a spacelike (resp. timelike, causal) segment $\left[\alpha\left(t_{j}\right), \alpha\left(t_{j+1}\right)\right]$ in a convex normal neighborhood of $\alpha\left(t_{j}\right)$. A continuous path $\alpha:[0,1] \rightarrow \mathcal{M}$ is said to be spacelike (resp. timelike, causal) if it has arbitrarily fine admissible spacelike (resp. timelike, causal) partitions. When this is the case, we can define the length of such a path as an infimum over all admissible partitions of the appropriate type:

$$
L(\alpha)=\inf \left\{L\left[\alpha\left(t_{0}\right), \alpha\left(t_{1}\right)\right]+\cdots+L\left[\alpha\left(t_{k-1}\right), \alpha\left(t_{k}\right)\right]\right\} .
$$

Note that it makes sense to define the length using the infimum versus the supremum, because $L$ satisfies the reverse triangle inequality, so refining a partition reduces the sum in (5.2).

The timelike separation of points $x, y \in \mathcal{M}$ is defined to be:

$$
\tau(x, y)=\sup \{L(\alpha) \mid \alpha \text { is a causal curve joining } x \text { and } y\}
$$

If there are no causal curves joining $x$ and $y$, then we set $\tau(x, y)=0$. One verifies easily that $\tau$ is symmetric and also satisfies the reverse triangle inequality.

For each $x \in \partial^{+} \mathbf{S}_{1}^{n}$ fix a future-pointing timelike geodesic $c$ with arclength parameter which converges to $x$ and define the timelike horofunction $\bar{\tau}_{x}: I^{-}(x) \rightarrow(0, \infty)$ by:

$$
\bar{\tau}_{x}(y)=\lim _{t \rightarrow \infty} \tau(y, c(t))-t .
$$


Flat conformal structures and the classification of de Sitter manifolds 335

Using the reverse triangle inequality, the expression on the right-hand side increases in $t$ and is bounded above, so the limit exists. The function sodefined is independent of the choice of $c$ up to an additive constant.

Now let $\Sigma$ denote a compact $n$-manifold without boundary, with a fixed flat conformal structure $\left(D_{\infty}, \phi\right) \in \mathcal{C}(\Sigma)$ of hyperbolic type, and space of strata $\mathcal{S}$ in $\tilde{\Sigma}$. We start the construction of the standard de Sitter spacetimes by defining a map $D_{0}^{*}: \mathcal{S} \rightarrow\left(\mathbf{H}^{n+1}\right)^{*}$. Recall that each stratum $s \in \mathcal{S}$ corresponds to a unique open round ball $U \subset \tilde{\Sigma}$; the set $\partial D_{\infty}(U)$ bounds a hyperplane in $\mathbf{H}^{n+1}$ which determines the desired point $D_{0}^{*}(s)$ in $\left(\mathbf{H}^{n+1}\right)^{*}$. Clearly nearby pairs of points in the image of this map are spacelike-separated, for if not, the open round ball corresponding to one of the points would be contained in the interior of the other (with perhaps one common boundary point) - this is impossible if each open round ball defines a non-empty stratum. It follows that any path in $\mathcal{S}$ maps to a continuous spacelike path in de Sitter space, and therefore has an induced length. This defines a metric space structure on $\mathcal{S}$.

Next note that there is a canonical map from $\tilde{\Sigma}$ to $\mathcal{S}$, given by $p \mapsto C\left(U_{p}\right)$; the composition with $D_{0}^{*}$ defines a map of $\tilde{\Sigma}$ into $\left(\mathbf{H}^{n+1}\right)^{*}$, which by abuse of notation we again denote $D_{0}^{*}$. Define a map $D^{*}: \tilde{\Sigma} \times(0, \infty) \rightarrow\left(\mathbf{H}^{n+1}\right)^{*}$ by sending $(p, t)$ to the point on the unique timelike ray from $D_{0}^{*}(p)$ to $D_{\infty}(p)$ satisfying $\tau\left(D_{0}^{*}(p), D^{*}(p, t)\right)=t$. This map can be lifted to $\mathbf{S}_{1}^{n+1}$ in such a way that as $t \rightarrow \infty$ the image approaches past infinity; we also write $D^{*}$ for the lifted map.

If $\Sigma$ is of parabolic type and $x \in \mathbf{S}^{n}$ is the point missed by $D_{\infty}$, then we can define $D^{*}: \tilde{\Sigma} \times(0, \infty) \rightarrow \mathbf{S}_{1}^{n+1}$ by sending $(p, t)$ to the point on the unique timelike ray from $x \in \partial_{\infty}^{+} \mathbf{S}_{1}^{n+1}$ to $D_{\infty}(p) \in \partial_{\infty}^{-} \mathbf{S}_{1}^{n+1}$ satisfying $\bar{\tau}_{x}\left(D^{*}(p, t)\right)=-\log t$ (since $\tau$ is only well-defined up to an additive constant, $D^{*}$ in this case is well-defined up to a multiplicative rescaling of $\left.(0, \infty)\right)$.

Finally, if $\Sigma$ is of elliptic type, then we use the homeomorphism of $\mathbf{S}_{1}^{n+1}$ with $\mathbf{S}^{n} \times \mathbf{R}$ (coming from its embedding in $\mathbf{R}_{1}^{n+2}$ ) to define $D^{*}$; as above we simply rescale $(0, \infty)$ by $t \mapsto-\log t$.

By an abuse of notation, the composition $\pi_{1}(\Sigma \times(0, \infty)) \cong \pi_{1}(\Sigma) \stackrel{\phi}{\rightarrow}$ $S O_{0}(n, 1)$ will also be denoted $\phi$.

Proposition 5.1. The pair $\left(D^{*}, \phi\right)$ defines a de Sitter structure on $\Sigma \times$ $(0, \infty)$ which is past complete. For every $t \in(0, \infty)$, the slice $\Sigma \times\{t\}$ is a global Cauchy hypersurface. 
Proof. We will first show that $D^{*}$ is a $\phi$-equivariant $C^{1}$ immersion. This is clear in the elliptic and parabolic cases; we may therefore restrict our attention to the case that $\Sigma$ is of hyperbolic type. It has already been remarked that the canonical stratification is equivariant, so given $\gamma \in \pi_{1}(\Sigma)$ and $p \in \tilde{\Sigma}$, we have $C\left(U_{\gamma \cdot p}\right)=\gamma \cdot C\left(U_{p}\right)$. The $\phi$-equivariance of $D_{\infty}$ then implies that $D_{0}^{*}$ and hence $D^{*}$ are also $\phi$-equivariant. The differentiability of $D^{*}$ can be proven by adapting the dual argument of Bowditch found in [7].

The proof that each slice is a global Cauchy hypersurface requires no further mention of the specific de Sitter structure involved. A slice $\Sigma \times\left\{t_{0}\right\}$ is a closed spacelike hypersurface by construction, and is clearly acausal since it is spacelike and separates $\Sigma \times(0, \infty)$. By Lemma 3.1, $D^{+}\left(\Sigma \times\left\{t_{0}\right\}\right) \cup$ $D^{-}\left(\Sigma \times\left\{t_{0}\right\}\right)$ is open, so it suffices to show that this set is also closed. We will show $H^{+}\left(\Sigma \times\left\{t_{0}\right\}\right)=H^{-}\left(\Sigma \times\left\{t_{0}\right\}\right)=\emptyset$. In light of Lemma 3.2, let $\beta$ be a null geodesic and define $L=\{t \in(0, \infty) \mid \beta \cap(\Sigma \times\{t\}) \neq \emptyset\}$. This set is nonempty and clearly open because each slice is spacelike and $\beta$ is a null curve. Suppose $\left\{t_{j}\right\}$ is a sequence of points in $L$ converging to some value $t \in(0, \infty)$; then by compactness of the slices, there is some point $z \in \Sigma \times\{t\}$ such that $\beta$ enters arbitrarily small convex normal neighborhoods of $z$. Again using the fact that the slices are spacelike, this forces $\beta$ to intersect $\Sigma \times\{t\}$, and so $L$ is closed. We conclude $L=(0, \infty)$, finishing the proof.

Proposition 5.1 provides a well-defined map $\Omega^{+}: \mathcal{C}(\Sigma) \rightarrow \mathrm{S}_{1}^{n+1}(\Sigma \times$ $(0, \infty))$. By reversing the time-orientations in each case (e.g. in the hyperbolic case, choosing the other possible lift of $D^{*}$ from $\left(\mathbf{H}^{n+1}\right)^{*}$ to $\mathbf{S}_{1}^{n+1}$ ), we obtain a second family of de Sitter structures and a map $\Omega^{-}: \mathcal{C}(\Sigma) \rightarrow \mathbf{S}_{1}^{n+1}(\Sigma \times(0, \infty))$. We say $\mathcal{M}$ is a standard de Sitter spacetime if $\mathcal{M} \approx \Sigma \times(0, \infty)$ and $\mathcal{M}$ is equipped with a de Sitter structure in $\Omega^{+}(\mathcal{C}(\Sigma)) \cup \Omega^{-}(\mathcal{C}(\Sigma))$. In this case, Proposition 5.1 also shows that for every $t \in(0, \infty)$, the slice $\Sigma \times\{t\}$ is a spacelike de Sitter hypersurface in the sense of $\S 3$. By construction, it is also clear that $\mathcal{M}_{\max }(\Sigma \times\{t\})=\Sigma \times(0, \infty)$. A standard de Sitter spacetime is said to be hyperbolic (resp. parabolic, elliptic) if it comes from a flat conformal structure of hyperbolic (resp. parabolic, elliptic) type.

There is a dual construction of a hyperbolic metric on $\Sigma \times(0, \infty)$ arising from a flat conformal structure on $\Sigma$. When $\Sigma$ is two-dimensional, this yields Thurston's parameterization of $\mathbf{C} P^{1}$-structures by measured geodesic laminations; the space of strata in this case is an $\mathbf{R}$-tree. These matters are discussed at length in [27] and [20]. 
Flat conformal structures and the classification of de Sitter manifolds 337

\section{Convexity Properties.}

Recall that a spacelike or timelike geodesic in a Lorentzian manifold may be parameterized in proportion to arclength in the usual way, while a natural choice of parameter for a null geodesic only exists up to an affine change of coordinates. The following lemma is the dual of an analogous statement for hyperbolic space; the proof itself is precisely dual to the one given by Douady in [6] for geodesics in $\mathbf{H}^{2}$.

Lemma 6.1. Suppose $\alpha:[0,1] \rightarrow \mathbf{S}_{1}^{n}$ and $\beta:[0,1] \rightarrow \mathbf{S}_{1}^{n}$ are spacelike or null segments with arclength or affine parameterizations such that for all $t \in[0,1]$ we have $\tau\left(\alpha_{t}, \beta_{t}\right)>0$. Then the function $t \mapsto-\tau\left(\alpha_{t}, \beta_{t}\right)$ is strictly convex.

Proof. We view all points of $\mathbf{S}_{1}^{n}$ as totally geodesic hyperplanes in $\mathbf{H}^{n}$. With this in mind, define $\sigma_{\alpha} \in O(n, 1)$ to be the reflection in the hyperplane $\alpha_{\frac{1}{2}}$; this isometry interchanges $\alpha_{0}$ and $\alpha_{1}$. Define $\sigma_{\beta}$ similarly, and let $\delta$ be the common perpendicular geodesic to $\alpha_{\frac{1}{2}}$ and $\beta_{\frac{1}{2}}$. Note that $\delta$ is invariant under both $\sigma_{\alpha}$ and $\sigma_{\beta}$, hence also under $\sigma_{\beta} \sigma_{\alpha}$. The critical remark is that the function $\tau\left(-, \sigma_{\beta} \sigma_{\alpha}-\right)$ on de Sitter space achieves its minimum precisely on those hyperplanes perpendicular to $\delta$ (equivalently, on the $(n-2)$-plane in de Sitter space dual to $\delta$ ). Thus:

$$
\begin{aligned}
\tau\left(\alpha_{1}, \beta_{1}\right)+\tau\left(\alpha_{0}, \beta_{0}\right) & =\tau\left(\alpha_{1}, \beta_{1}\right)+\tau\left(\sigma_{\alpha} \alpha_{1}, \sigma_{\beta} \beta_{1}\right) \\
& =\tau\left(\alpha_{1}, \beta_{1}\right)+\tau\left(\sigma_{\beta} \sigma_{\alpha} \alpha_{1}, \beta_{1}\right) \\
& \leq \tau\left(\alpha_{1}, \sigma_{\beta} \sigma_{\alpha} \alpha_{1}\right) \\
& <\tau\left(\alpha_{\frac{1}{2}}, \sigma_{\beta} \sigma_{\alpha} \alpha_{\frac{1}{2}}\right) \\
& =\tau\left(\alpha_{\frac{1}{2}}, \sigma_{\beta} \alpha_{\frac{1}{2}}\right) \\
& =\tau\left(\alpha_{\frac{1}{2}}, \beta_{\frac{1}{2}}\right)+\tau\left(\beta_{\frac{1}{2}}, \sigma_{\beta} \alpha_{\frac{1}{2}}\right) \\
& =2 \tau\left(\alpha_{\frac{1}{2}}, \beta_{\frac{1}{2}}\right) .
\end{aligned}
$$

A hypersurface $\Sigma$ in a de Sitter spacetime is locally convex from the future if at every point $x \in \Sigma$ there is a null or spacelike support plane such that a neighborhood of $x$ in $\Sigma$ lies on or in the past of the support plane. Similarly, $\Sigma$ is locally strictly convex from the future if the support planes 
meet $\Sigma$ locally in a single point. The application of Lemma 6.1 which we will need is the following:

Proposition 6.2. Let $\Sigma$ be a spacelike de Sitter hypersurface identified with its image in $\mathcal{M}_{\max }(\Sigma)$. Suppose that $H^{+}(\tilde{\Sigma}) \subset \overline{\mathcal{M}}_{\text {max }}(\tilde{\Sigma})$ is non-empty and locally convex from the future, with degenerate support planes corresponding to the null generators of $H^{+}(\tilde{\Sigma})$. Then a neighborhood in the past of $H^{+}(\tilde{\Sigma})$ is foliated by global Cauchy hypersurfaces for $\mathcal{M}_{\max }(\tilde{\Sigma})$ which are locally strictly convex from the future and which project to global Cauchy hypersurfaces for $\mathcal{M}_{\max }(\Sigma)$.

Proof. We begin by assuming that $\Sigma$ is simply-connected, and so $H^{+}(\Sigma)$ has the properties guaranteed by Proposition 3.3. Define the time-to-horizon function $\tau_{H^{+}}: D^{+}(\Sigma) \rightarrow(0,+\infty]$ by setting

$$
\tau_{H^{+}}(x)=\sup \left\{\tau(x, y) \mid y \in H^{+}(\Sigma)\right\} .
$$

We claim that if $\tau_{H^{+}}(x)=+\infty$ at any point $x \in D^{+}(\Sigma)$, then $\tau_{H^{+}} \equiv+\infty$ on all of $D^{+}(\Sigma)$. The set of points where $\tau_{H^{+}}$equals infinity is clearly open, so consider a point $x_{0}$ such that $\tau_{H^{+}}\left(x_{0}\right)<+\infty$. The future-pointing timelike rays from $x_{0}$ all meet $H^{+}(\Sigma)$ in finite time, so by the local convexity of $H^{+}(\Sigma)$ the same holds for the future-pointing null rays from $x_{0}$. There exist local spacelike or null support planes at these intersection points, which extend slightly outside of $\overline{I^{+}\left(x_{0}\right)}$. This forces $\tau_{H^{+}}<+\infty$ on a neighborhood of $x_{0}$, proving the claim.

So now suppose $\tau_{H^{+}} \equiv+\infty$ on all of $D^{+}(\Sigma)$. It follows that every null generator of $H^{+}(\Sigma)$ is future complete; for if some null generator $\lambda$ had a future endpoint $p \in H^{+}(\Sigma)$, then we could find a spacelike local support plane at $p$, which would force points in a small enough neighborhood in the past of $p$ to satisfy $\tau_{H^{+}}<+\infty$. These points lie in $D^{+}(\Sigma)$ however, a contradiction. Given a future complete null generator $\lambda$, let $N$ be the unique degenerate hyperplane containing $\operatorname{dev}(\lambda)$; this is a future local support plane for the image of $H^{+}(\Sigma)$ by hypothesis. But if a point of $H^{+}(\Sigma)$ near $\lambda$ develops to the past of $N$, we can apply the time reverse of Proposition 3.4 to see $I^{-}(N)=I^{-}(\operatorname{dev}(\lambda))$. Thus we can find a past-pointing timelike curve in $\mathcal{M}$ joining two points of $H^{+}(\Sigma)$; this contradicts the achronality of $H^{+}(\Sigma)$. We conclude that the entire connected component of $H^{+}(\Sigma)$ containing $\lambda$ develops into $N$, and so we can take as our global Cauchy hypersurfaces the level sets of a timelike horofunction for the future endpoint $z$ of $\operatorname{dev}(\lambda)$ on $\partial_{\infty}^{+} \mathbf{S}_{1}^{n}$. One checks easily that these surfaces are locally strictly convex from 
Flat conformal structures and the classification of de Sitter manifolds 339

the future (they are, in fact, dual to the horospheres based at $z \in \partial_{\infty} \mathbf{H}^{n}$, which are clearly strictly convex).

Finally, we may assume that $\tau_{H^{+}}<+\infty$ on all of $D^{+}(\Sigma)$. In this case there is a continuous "farthest-point retraction" $r: D^{+}(\Sigma) \rightarrow H^{+}(\Sigma)$; the proofs of existence and continuity are dual to the analogous proofs for hyperbolic space which can be found in [7]. We claim that the level sets $\tau_{H^{+}}^{-1}(t)$ for small values of $t$ foliate a neighborhood in the past of $H^{+}(\Sigma)$ and are locally strictly convex from the future.

Take $x \neq y$ to be two points in $\tau_{H^{+}}{ }^{-1}[\epsilon,+\infty)$ which are spacelikeseparated and close enough so that $r(x)$ and $r(y)$ lie in a locally convex neighborhood on $H^{+}(\Sigma)$. Let $\alpha:[0,1] \rightarrow \mathbf{S}_{1}^{n}$ be a spacelike segment joining $x$ to $y$, with arclength parameter. Similarly, let $\beta$ be a (possibly null) segment joining $r(x)$ to $r(y)$. It follows from Lemma 6.1 that

$$
\epsilon \leq \frac{1}{2}(\tau(\alpha(0), \beta(0))+\tau(\alpha(1), \beta(1)))<\tau\left(\alpha\left(\frac{1}{2}\right), \beta\left(\frac{1}{2}\right)\right) ;
$$

therefore if $\beta\left(\frac{1}{2}\right) \in H^{+}(\Sigma)$ we are done, otherwise continue the future pointing segment from $\alpha\left(\frac{1}{2}\right)$ through $\beta\left(\frac{1}{2}\right)$ to $H^{+}(\Sigma)$ to complete the proof for $\Sigma$ simply-connected.

Finally, when $\Sigma$ is not simply-connected, we perform the construction above for $\tilde{\Sigma}$ and note that each step is equivariant with respect to the covering transformations.

Proposition 6.3. If $\Sigma \times(0, \infty)$ is a hyperbolic or parabolic standard de Sitter spacetime, then for every $t \in(0, \infty)$ the slice $\Sigma \times\{t\}$ is locally strictly convex.

Proof. It was noted in the proof of Proposition 6.2 that the slices in a parabolic standard de Sitter spacetime are dual to horospheres in hyperbolic space and are therefore locally strictly convex. Suppose therefore that $\Sigma \times(0, \infty)$ is a hyperbolic standard de Sitter spacetime, and fix $t \in(0, \infty)$.

It was remarked above that $\mathcal{M}_{\max }(\Sigma \times\{t\})=\Sigma \times(0, \infty)$; The Cauchy horizon $H^{+}(\tilde{\Sigma} \times\{t\})$ in $\overline{\mathcal{M}}_{\max }(\tilde{\Sigma} \times\{t\})$ is in one-to-one correspondence with the set of open round balls $U \subset \tilde{\Sigma}$ such that $U_{\infty} \neq \emptyset$. One sees that $H^{+}(\tilde{\Sigma} \times\{t\})$ satisfies the hypotheses of Proposition 6.2 in the following manner. Let $U$ be an open round ball with $p \in U_{\infty}$; the failure of the local convexity property translates into the existence of a nearby open round ball which contains $p$, contradicting the fact that $p \in U_{\infty}$. The proposition is then a corollary of the previous proof, as the locally strictly convex surfaces 
constructed there are precisely the hypersurfaces $\tilde{\Sigma} \times\{t\}$ of constant timelike separation $t$ from $H^{+}(\tilde{\Sigma} \times\{t\})$.

We shall see, in fact, that the proof given above of the locally convexity of of the Cauchy horizon for a standard de Sitter spacetime works quite generally; this will allow us to obtain the Classification Theorem 1.1 in the next section.

\section{Proofs of Main Theorems.}

The first proposition is the key observation from which our main result is derived.

Proposition 7.1. Suppose $\mathcal{M}$ is a compact de Sitter spacetime, and $\Sigma \subset$ $\mathcal{M}$ is a closed acausal spacelike hypersurface. Suppose further that $H^{+}(\Sigma)$ is non-empty, and the null generators of $H^{+}(\Sigma)$ are past complete. Then $H^{+}(\Sigma)$ is locally convex from the future, with degenerate support planes corresponding to the null generators of $H^{+}(\Sigma)$.

Proof. Write dev $: \tilde{\mathcal{M}} \rightarrow \mathrm{S}_{1}^{n}$ for the developing map. Take $x \in H^{+}(\Sigma)$; by Lemma 3.2 there exists an inextendible, past-pointing null generator $\beta$ starting at $x$ and lying entirely within $H^{+}(\Sigma)$. Choose a lift $\tilde{x} \in \tilde{\mathcal{M}}$ of $x$, and let $\tilde{\beta}$ be the lift of $\beta$ starting at $\tilde{x}$. There is a unique degenerate hyperplane $N \subset \mathrm{S}_{1}^{n}$ containing $\operatorname{dev}(\tilde{\beta})$; we claim $N$ is a local support plane for the developing image of a neighborhood of $\tilde{x}$ in $H^{+}(\tilde{\Sigma})=\widetilde{H^{+}(\Sigma)}$. Suppose not, so there exist points $p \in I^{+}(N)$ in the developing image of arbitrarily small neighborhoods of $\tilde{x}$ in $H^{+}(\tilde{\Sigma})$. But Proposition 3.4 implies that $p \in$ $I^{+}(\operatorname{dev}(\tilde{\beta}))$; choosing $p$ close enough to $\operatorname{dev}(\tilde{x})$, we assure the existence of a past-pointing timelike curve from $p$ to a point of $\operatorname{dev}(\tilde{\beta})$ which lies entirely within the developing image of a small neighborhood of $\tilde{\beta}$. This contradicts the fact that $H^{+}(\Sigma)$ is achronal, proving the proposition.

We have modified the statement of Theorem 1.1, taking advantage of the formalism of $\S 3$ :

Theorem 7.2. If $\Sigma$ is a spacelike de Sitter hypersurface, then $\mathcal{M}_{\text {max }}(\Sigma)$ is a standard de Sitter spacetime. 
Flat conformal structures and the classification of de Sitter manifolds 341

Proof. If $\mathcal{M}_{\text {max }}(\Sigma)$ is both past and future complete, it follows that it is isometric to a manifold of the form $\mathbf{S}_{1}^{n} / \Gamma$ for some finite subgroup of $\Gamma$ of $S O_{0}(n, 1)$. It is well-known $[30,11.2]$ that all such subgroups are conjugate into the maximal compact subgroup $S O(n)$ of $S O_{0}(n, 1)$, and therefore $\Gamma$ acts freely and isometrically on $\partial_{\infty}^{+} \mathbf{S}_{1}^{n+1}$ and $\partial_{\infty}^{-} \mathbf{S}_{1}^{n+1}$. Thus $\mathcal{M}_{\max }(\Sigma)$ is a standard de Sitter spacetime arising from the spherical space form sodefined.

Assume now, without loss of generality, that $\mathcal{M}_{\max }(\Sigma)$ fails to be future complete and apply Proposition 3.3 to embed $\mathcal{M}_{\text {max }}(\tilde{\Sigma})$ in $\overline{\mathcal{M}}_{\text {max }}(\tilde{\Sigma})$ so that $H^{+}(\tilde{\Sigma}) \neq \emptyset$ and all null generators are past complete. Combining Propositions 7.1 and 6.2 shows that there is a global Cauchy hypersurface $\Sigma^{\prime}$ for $\mathcal{M}_{\max }(\Sigma)$ which is locally strictly convex from the future. Thus $\mathcal{M}_{\max }(\Sigma) \subseteq \mathcal{M}_{\max }\left(\Sigma^{\prime}\right)$. Under this inclusion $\Sigma$ becomes a global Cauchy hypersurface for the spacetime $\mathcal{M}_{\max }\left(\Sigma^{\prime}\right)$ since any causal curve in $\mathcal{M}_{\text {max }}\left(\Sigma^{\prime}\right)$ meets $\Sigma^{\prime}$ and therefore $\Sigma$ also; hence $\mathcal{M}_{\max }(\Sigma)=\mathcal{M}_{\max }\left(\Sigma^{\prime}\right)$.

Because $\Sigma^{\prime}$ is locally strictly convex and spacelike, we can define a corresponding flat conformal structure on $\Sigma^{\prime}$ by the Gauss map; i.e. following the unique timelike normal line at each point of the developing image of $\tilde{\Sigma}^{\prime}$ to past infinity defines an equivariant developing map $D_{\infty}: \tilde{\Sigma}^{\prime} \rightarrow \partial_{\infty}^{-} \mathbf{S}_{1}^{n+1}$. The standard de Sitter spacetime corresponding to this flat conformal structure contains $\Sigma^{\prime}$ as a global Cauchy hypersurface, and so it equals $\mathcal{M}_{\max }\left(\Sigma^{\prime}\right)$. Thus $\mathcal{M}_{\text {max }}(\Sigma)$ is a standard de Sitter spacetime.

\section{Horizons in Standard de Sitter Spacetimes.}

Our main theorem classifies compact de Sitter domains of dependence by flat conformal structures arising at timelike infinity. In [23], it is shown that every compact flat or anti-de Sitter spacetime-bordism is a domain of dependence (in particular, a topological product $\Sigma \times \mathbf{R}$ with spacelike slices) by modifying the arguments of [4]. The analogous result is not quite true in the de Sitter case, as there are simple counterexamples in dimension two. It follows from Euler characteristic considerations that any two-dimensional spacetime-bordism is homeomorphic to an annulus; nevertheless we have found a family of de Sitter annuli which contain non-trivial Cauchy horizons. 


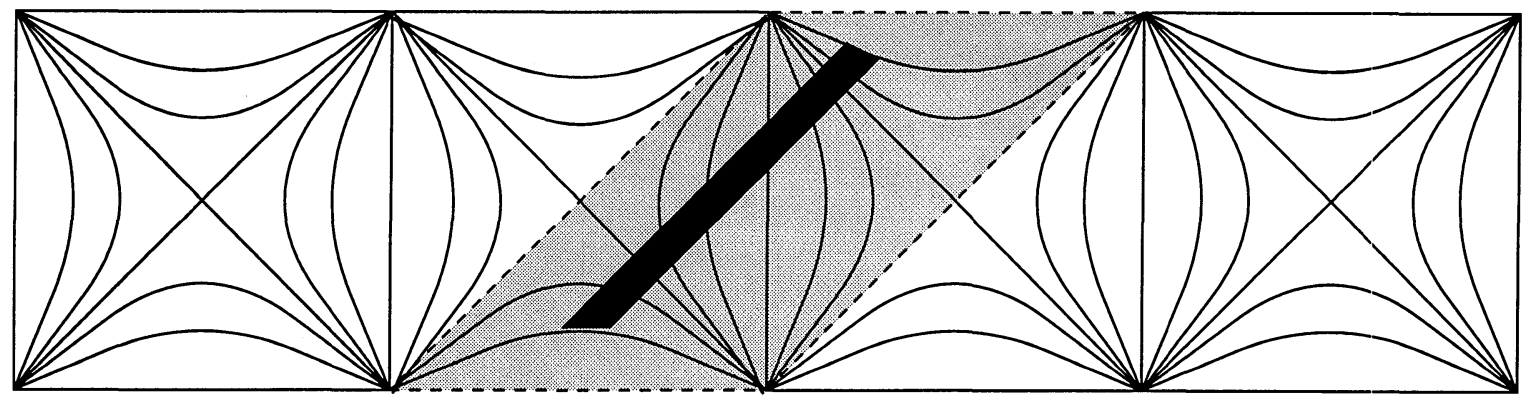

Figure 1: The lightly shaded region indicates the universal cover of an open $1+1$ de Sitter annulus with hyperbolic holonomy which is not a domain of dependence. The darker region is a fundamental domain for compact annulus with spacelike boundary.

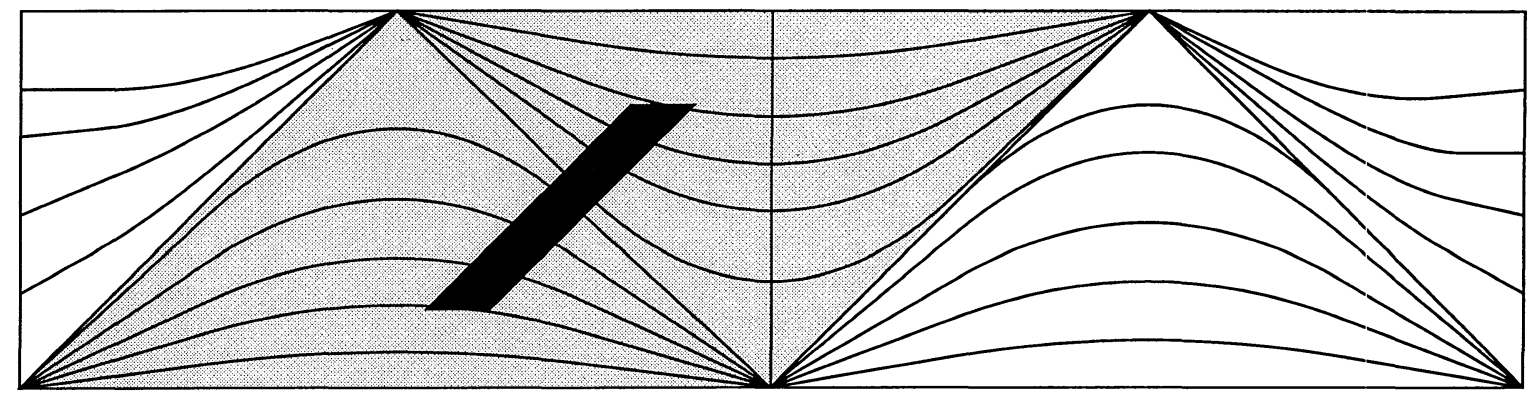

Figure 2: The lightly shaded region indicates the universal cover of an open $1+1$ de Sitter annulus with parabolic holonomy which is not a domain of dependence. The darker region is a fundamental domain for compact annulus with spacelike boundary and no closed timelike curves.

Suppose $\tau \in S O_{0}(2,1)$ is a hyperbolic element, and fix a ruling of $\mathbf{S}_{1}^{2}$ by null lines. This defines a pair of disjoint null lines in $\mathbf{S}_{1}^{2}$ joining the two fixed points of $\tau$ in $\partial_{\infty}^{+} \mathbf{S}_{1}^{2}$ with the ones in $\partial_{\infty}^{-} \mathbf{S}_{1}^{2}$. The element $\tau$ acts freely and properly discontinuously on an open region $\mathcal{U}$ bounded by these two lines (compare figure 1, where for simplicity we have indicated this situation in the universal cover of $\mathbf{S}_{1}^{2}$ ). The quotient $\mathcal{U} /\langle\tau\rangle$ is a de Sitter annulus with two non-trivial Cauchy horizons corresponding to the two null rays in $\mathcal{U}$ left invariant by $\tau$. One can construct similar examples which contain no closed 
Flat conformal structures and the classification of de Sitter manifolds 343

timelike curves by choosing $\tau$ to be parabolic. See figure 2 .

In dimension three, similar examples arise when there are open subsets in the Cauchy horizon which are foliated by null generators (arising from Hopf submanifolds in the flat conformal structure at infinity) It is shown in [27] that these are the only possibilities. There is presumably a similar result in higher dimensions, which will require a more careful analysis of the group action on the space of maximal balls.

\section{References.}

[1] B.N. Apanasov, The geometry of Nielsen's hull for a Kleinian group in space and quasi-conformal mappings, Ann. Global Anal. Geom. 6 (1988), no. 3, 207-230.

[2] J.K. Beem, P.E. Ehrlich, and K.L. Easley, Global Lorentzian geometry, 2 ed., Monogr. Textbooks Pure Appl. Math, vol. 202, Marcel Dekker, Inc., New York, 1996.

[3] R.D. Canary, D.B.A. Epstein, and P. Green, Notes on notes of Thurston, Analytical and Geometric Aspects of Hyperbolic Space (D.B.A. Epstein, ed.), London Math. Soc. Lecture Note Ser., vol. 111, Cambridge Univ. Press, Cambridge, 1987, pp. 3-92.

[4] Y. Carrière, Autour de la conjecture de L. Markus sur les variétés affines, Invent. Math. 95 (1989), 615-628.

[5] R.M. Charney and M.W. Davis, The polar dual of a convex polyhedral set in hyperbolic space, Michigan Math. J. 42 (1995), no. 3, 479-510.

[6] A. Douady, L'espace de Teichmüller, Travaux de Thurston sur les surfaces (A. Fathi, F. Laudenbach, and V. Poénaru, eds.), Astérisque, vol. 66, Soc. Math. France, Paris, 2 ed., 1979, pp. 127-137.

[7] D.B.A. Epstein and A. Marden, Convex hulls in hyperbolic space, a theorem of Sullivan, and measured pleated surfaces, Analytical and Geometric Aspects of Hyperbolic Space (D.B.A. Epstein, ed.), London Math. Soc. Lecture Note Ser., vol. 111, Cambridge Univ. Press, Cambridge, 1987, pp. 113-254.

[8] D.L. Fried and W.M. Goldman, Three-dimensional affine crystallographic groups, Adv. Math. 47 (1983), 1-49.

[9] R.P. Geroch, Domain of dependence, J. Math. Phys. 11 (1970), no. 2, 437-449.

[10] W.M. Goldman, Nonstandard Lorentz space forms, J. Differential Geom. 21 (1985), 301-308. 
[11] Geometric structures on manifolds and varieties of representations, Geometry of Group Representations (W.M. Goldman and A.R. Magid, eds.), Contemp. Math., vol. 74, Amer. Math. Soc., Providence, 1988, pp. 169197.

[12] W.M. Goldman and Y. Kamishima, The fundamental group of a compact flat Lorentz space form is virtually polycyclic, J. Differential Geom. 19 (1984), 233-240.

[13] S.G. Harris, What is the shape of space in a spacetime?, Differential geometry: geometry in mathematical physics and related topics, Proc. Sympos. Pure Math., vol. 2, Amer. Math. Soc., Providence, 1993, pp. 287-296.

[14] S.W. Hawking and G.F.R. Ellis, The large scale structure of space-time, Cambridge Univ. Press, Cambridge, 1973.

[15] C.D. Hodgson and I.Rivin, A characterization of compact convex polyhedra in hyperbolic 3-space, Invent. Math. 111 (1993), no. 1, 77-111.

[16] Y. Kamishima and S.P. Tan, Deformation spaces on geometric structures, Aspects of low-dimensional manifolds (Y. Matsumoto and S. Morita, eds.), Adv. Stud. Pure Math., vol. 20, Kinokuniya, Tokyo, 1992, pp. 263-299.

[17] B. Klingler, Complétude des variétés Lorentziennes à courbure constante, Math. Ann. 306 (1996), no. 2, 353-370.

[18] R.S. Kulkarni, Conformal structures and Möbius structures, Conformal Geometry (R.S. Kulkarni and U. Pinkall, eds.), Aspects Math., vol. 12, Vieweg, Braunschweig, 1988, pp. 1-39.

[19] R.S. Kulkarni and U. Pinkall, Uniformization of geometric structures with applications to conformal geometry, Differential Geometry, Peñiscola, 1985 (A.M. Naveira, A. Ferrández, and F. Mascaró, eds.), Lecture Notes in Math., vol. 1209, Springer-Verlag, New York-Berlin-Heidelberg, 1986, pp. 190-209.

[20] A canonical metric for Möbius structures and its applications, Math. Z. 216 (1994), 89-129.

[21] R.S. Kulkarni and F.A. Raymond, 3-dimensional Lorentz space-forms and Seifert fiber spaces, J. Differential Geom. 21 (1985), 231-268.

[22] S. Matsumoto, Foundations of flat conformal structure, Aspects of lowdimensional manifolds (Y. Matsumoto and S. Morita, eds.), Adv. Stud. Pure Math., vol. 20, Kinokuniya, Tokyo, 1992, pp. 167-261.

[23] G. Mess, Lorentz spacetimes of constant curvature, MSRI Preprint 90-05808, 1990. 
Flat conformal structures and the classification of de Sitter manifolds 345

[24] M.M. Morrill, Non-existence of compact de Sitter manifolds, Ph.D. thesis, University of California, Los Angeles, 1997.

[25] B. Ó Neill, Semi-Riemannian Geometry, Academic Press, New York-London, 1983.

[26] V.I. Oliker, A priori estimates of the principal curvatures of spacelike hypersurfaces in de Sitter space with applications to hypersurfaces in hyperbolic space, Amer. J. Math. 114 (1992), no. 3, 605-626.

[27] K.P. Scannell, Flat conformal structures and causality in de Sitter manifolds, Ph.D. thesis, University of California, Los Angeles, 1996.

[28] W.P. Thurston, The Geometry and Topology of Three-Manifolds, Princeton Univ., Princeton, 1982.

[29] E. Witten, $2+1$ dimensional gravity as an exactly soluble system, Nuclear Phys. B 311 (1989), 46-78.

[30] J.A. Wolf, Spaces of Constant Curvature, 5 ed., Publish or Perish, Inc., Houston, 1984.

RECEIVED FEBRUARY 10, 1997.

Department of Mathematics and Computer Science

SAINT LOUIS UNIVERSITY

SAINT Louis, MO 63103

E-mail address: scannell@slu.edu 